\title{
Effect of the In-Cylinder Back Pressure on the Injection Process and Fuel Flow Characteristics in a Common-Rail Diesel Injector Using GTL Fuel
}

\author{
Luka Lešnik ${ }^{1}$, Breda Kegl ${ }^{1}$, Eloísa Torres-Jiménez ${ }^{2} \oplus$, Fernando Cruz-Peragón ${ }^{2}$, Carmen Mata ${ }^{3, *}$ and Ignacijo Biluš ${ }^{1}$ \\ 1 Faculty of Mechanical Engineering, University of Maribor, Smetanova ulica 17, SI-2000 Maribor, Slovenia; \\ luka.lesnik@um.si (L.L.); breda.kegl@um.si (B.K.); ignacijo.bilus@um.si (I.B.) \\ 2 Department of Mechanical and Mining Engineering, Campus las Lagunillas, University of Jaén, s/n, \\ 23071 Jaén, Spain; etorres@ujaen.es (E.T.-J.); fcruz@ujaen.es (F.C.-P.) \\ 3 Escuela de Ingeniería Minera e Industrial de Almadén, Campus de Excelencia Internacional en Energía y \\ Medioambiente, Universidad de Castilla-La Mancha, Plaza Meca s/n, 13400 Almadén, Spain \\ * Correspondence: mariacarmen.mata@uclm.es; Tel.: +349-2629-5300 (ext. 6042)
}

Citation: Lešnik, L.; Kegl, B.;

Torres-Jiménez, E.; Cruz-Peragón, F.; Mata, C.; Biluš, I. Effect of the In-Cylinder Back Pressure on the Injection Process and Fuel Flow Characteristics in a Common-Rail Diesel Injector Using GTL Fuel.

Energies 2021, 14, 452.

https://doi.org/10.3390/en14020452

Received: 18 December 2020

Accepted: 13 January 2021

Published: 15 January 2021

Publisher's Note: MDPI stays neutral with regard to jurisdictional claims in published maps and institutional affiliations.

Copyright: (C) 2021 by the authors Licensee MDPI, Basel, Switzerland. This article is an open access article distributed under the terms and conditions of the Creative Commons Attribution (CC BY) license (https:/ / creativecommons.org/licenses/by/ $4.0 /)$.

\begin{abstract}
The presented paper aims to study the influence of mineral diesel fuel and synthetic Gas-ToLiquid fuel (GTL) on the injection process, fuel flow conditions, and cavitation formation in a modern common-rail injector. First, the influence on injection characteristics was studied experimentally using an injection system test bench, and numerically using the one-dimensional computational program. Afterward, the influence of fuel properties on internal fuel flow was studied numerically using a computational program. The flow inside the injector was considered as multiphase flow and was calculated through unsteady Computational Fluid Dynamics simulations using a EulerianEulerian two-fluid approach. Finally, the influence of in-cylinder back pressure on the internal nozzle flow was studied at three distinctive back pressures. The obtained numerical results for injection characteristics show good agreement with the experimental ones. The results of 3D simulations indicate that differences in fuel properties influence internal fuel flow and cavitation inception. The location of cavitation formation is the same for both fuels. The cavitation formation is triggered regardless of fuel properties. The size of the cavitation area is influenced by fuel properties and also from in-cylinder back pressure. Higher values of back pressure induce smaller areas of cavitation and vice versa. Comparing the conditions at injection hole exit, diesel fuel proved slightly higher average mass flow rate and velocities, which can be attributed to differences in fluid densities and viscosities. Overall, the obtained results indicate that when considering the injection process and internal nozzle flow, GTL fuel can be used in common-rail injection systems with solenoid injectors.
\end{abstract}

Keywords: nozzle flow; cavitation; multiphase flow; synthetic fuel; diesel injection; transient simulations

\section{Introduction}

The transport sector in the European Union (EU) consumes one-third of the total primary energy and accounts for one-fifth of total EU Carbon Oxide emissions [1,2]. The combustion of petroleum-based fuels powers most of today's transport sector and is mainly responsible for the high pollution in this sector [3]. The use of biofuels is considered a medium to a long-term alternative to petroleum-based fuels, which can reduce harmful emissions from the transport sector. Most of the biofuels used in the EU are mixed with conventional fuels in smaller ratios. Their usage is also promoted by the European Union, which predicts that, in $2020,10 \%$ of petroleum-based fuels should be replaced by biofuels [4]. The first generation of biofuels produced from rapeseed, cooking oil, animal fat, etc., represented the majority of biodiesel used in the EU in 2016 [5]. In the recent decade, a lot of research work was done on second-generation biofuels, which are compared to first-generation biofuels produced from non-edible biomass sources [6]. Much 
investigation has also focused on the development of synthetic fuels [7]. Their usage in compression ignition engines can also contribute to emission reduction in the transport sector [8] and increase the combustion efficiency [9].

The efficiency of combustion in modern diesel engines depends heavily on the fuel injection parameters, the fuel atomization process, the fuel properties and other related parameters. In order to improve those processes, injection pressure in modern compression ignition engines was increased to $2000-2500 \mathrm{bar}$, and multiple injection processes per cycle are being used [10-12]. These strategies resulted in an increased number of injection holes with a smaller diameter, which, combined with high injection pressure, can create flow conditions that promote cavitation formation. The cavitation can have a negative effect on fuel injector parts since it induces cavitation erosion. Nevertheless, it can be beneficial for the fuel spray atomization process [13-16].

In recent decades, numerical models have undergone great development. Using modern, numerical programs, experimental testing can be replaced with numerical experiments in several research and engineering fields [17].

The influence of fuel properties and engine operating conditions on in-nozzle cavitation and the spray break-up processes can be studied experimentally and/or numerically. Due to its tiny dimensions, experimental observations of flow characteristics inside the injector nozzle are usually performed using large scale optical nozzle models. These models allow easier observation, but the results can differ from real-sized injector nozzles $[18,19]$. In these studies, the similarity flow in real size and scale-up nozzles is usually achieved based on dynamic flow similarity based simultaneous on the Reynolds and cavitation number. The problem in large scale models is with cavitation phenomena at the microscale of cavitation bubble dynamics (bubble formation and development). Since this effect is related to the number of cavitation bubbles which further coalesce and form the cavitation structures (clouds), it is dependent on nozzle size and can differ in real size nozzles and large scale nozzles (models). The X-ray phase-contrast imaging technique and X-ray tracer imaging methods can be used to monitor real conditions during the injection process using petroleum-based fuels [20]. All the presented experimental methods are appropriate for monitoring flow conditions and cavitation inside the injector nozzle, but they are usually related to high costs of experimental equipment and long measurement procedures in the case of studying several fuels and operating regimes.

These disadvantages can be overcome by the use of 3D numerical simulations, which are performed using Computational Fluid Dynamic (CFD) programs like ANSYS CFX, ANSYS FLUENT, OpenFOAM, AVL FIRE, etc. Similar to X-ray imaging methods, these programs allow us to determine fuel flow conditions like velocity, cavitation inception, etc., in a non-intrusive way. When using numerical simulation, RANS, LES, or DNS approaches can be used [21]. Simulations can be performed at fixed or transient needle positions. Since the injection times are very short, the transient needle movement and transient conditions inside the injector are dominant. Needle movement during the injection process should be considered in the simulations to predict the transient conditions accurately. Numerical methods have been used and validated as a promising tool for studying flow conditions with cavitation inception in a fuel injector in several studies [12,13,21-25].

Modern compression ignition engines are very complex systems. Before using a new fuel or biofuel as a substitute or as an additive to conventional fuels, extensive testing must be done in several research and development areas, since differences in fuel properties can influence fuel injection processes, fuel combustion processes, obtained engine characteristics, and emission formation [26,27]. The presented work focuses on the research area of fuel injection.

Several studies have also discussed the influence of fuel properties on the cavitation formation inside the injection nozzle and on the conditions at the injection hole outlet. Yu et al. [28] numerically studied the influence of gasoline, diesel, wide distillation fuels, and kerosene on internal nozzle flow, spray formation, combustion, and emissions characteristic. The numerical studies of internal nozzle flow were made for one nozzle 
injection hole using the RANS approach at a constant needle position and different injection pressures. From the obtained results, they concluded that a lower fuel kinematic viscosity leads to higher values of Reynolds number, which causes higher turbulent kinetic energy and higher velocity of fuel flow. This correlation between lower viscosity and higher fuel velocity was also found in the study of Battistoni et al. [23], where the influence of Soybean oil methyl ester biodiesel and diesel on inner nozzle flow and spray atomization was studied. Again, the RANS approach was used for studying the conditions inside the injector, but transient needle movement of Bosch CRI1 common-rail injector was considered. Torelli et al. [29] used the RANS approach with the standard k- $\varepsilon$ turbulence model and tested the behavior of two gasoline-like fuels, and n-Dodecane fuel in internal nozzle flow at constant injection pressure and transient needle lifts. They also concluded that lower viscosities promote higher fuel velocities. Higher fuel velocities lead to an increase in fuel mass flow rate inside the injector or at the injector outlet [23,28-30]. Rapid changes in fuel flow and high fuel velocities significantly influence pressure variations inside the injector. This can cause the immediate pressure to drop to almost $0 \mathrm{~Pa}$, which lowers the impact of different fuels saturation pressures on cavitation formation [23]. The amount of cavitation fraction also depends on the difference between injection and in-cylinder pressure [28].

This brief literature review shows that no extensive studies were made about the influence of GTL fuel and its properties on conditions inside the injector. We also did not find any studies regarding the influence of in-cylinder back pressure on GTL fuel flow conditions and fuel atomization.

To fill this gap, the objectives in this study are to study the influence of GTL fuel properties on the injection process, fuel flow conditions, and cavitation inception in a modern common-rail injector. The study was performed on a Denso solenoid injector taken from a commercial NISSAN YD1-K2-Type engine. Conventional mineral diesel fuel and synthetic GTL fuel, produced from natural gas, were tested. Their influence on the injection process was tested experimentally and numerically using the 1D AVL Hydsim program at 60 bar of in-cylinder back pressure. The influence on cavitation inception and fuels flow conditions was tested using a 3D computational program AVL FIRE. Using this program, transient simulations, considering needle movement during injection of each fuel, have been carried through the RANS approach, standard mathematical, and turbulence models implemented in commercial software. Different ambient pressures of 40,60, and 80 bar were applied at the injection nozzle outlet in order to extend the work and study the effect of in-cylinder back pressure on conditions inside the injection nozzle.

The obtained results indicate that differences in GTL fuel properties and in-cylinder back pressures influence flow conditions and cavitation inception inside and at the outlet of the common-rail solenoid injector. These differences can have a potential influence on further processes in the combustion chamber of direct injection diesel engines. The possible influence of GTL fuel on fuel atomization, combustion, and emission formation will be discussed in the conclusion section.

\section{Tested Fuels}

Two different fuels were used in the presented paper. Neat diesel fuel was supplied by REPSOL Co., (REPSOL, Madrid and Spain) which did not contain any biodiesel, denoted as D2. GTL fuel was provided by SASOL Co. (SASOL Limited, Johannesburg and South Africa) and was produced from natural gas through the Fischer Tropsch process at a low temperature, denoted as GTL. The tested fuel properties are presented in Table 1. 
Table 1. Tested fuel properties [31].

\begin{tabular}{ccc}
\hline Properties & Diesel & GTL \\
\hline Density at $15^{\circ} \mathrm{C}\left(\mathrm{kg} / \mathrm{m}^{3}\right)$ & 813 & 771.4 \\
Kinematic Viscosity at $40^{\circ} \mathrm{C}(\mathrm{cSt})$ & 2.01 & 2.57 \\
Lubricity $(\mu \mathrm{m})$ & 334 & 353 \\
Lower calorific value $(\mathrm{MJ} / \mathrm{kg})$ & 43.13 & 43.86 \\
$\mathrm{C}(\% w / w)$ & 85.74 & 84.82 \\
$\mathrm{H}(\% w / w)$ & 14.26 & 15.18 \\
$\mathrm{O}(\% w / w)$ & 0 & 0 \\
Molecular weight $(\mathrm{g} / \mathrm{mol})$ & 187.4 & 238.9 \\
Cetane number & 65.36 & $>73$ \\
Saturation pressure $(\mathrm{Pa})$ & 1000 & 200 \\
Density at $15^{\circ} \mathrm{C}\left(\mathrm{kg} / \mathrm{m}^{3}\right)$ & 813 & 771.4 \\
\hline
\end{tabular}

\section{Experimental Set-Up}

This section presents the experimental setup and the methods used to obtain the experimental data. A solenoid operated Denso injector model 7H150 with 7 injection holes has been used in this work. All injection holes were the same, each with $150 \mu \mathrm{m}$ diameter and $760 \mu \mathrm{m}$ length. An X-ray scanner NIKON model CT-SCAN-XT-H-160 has been used to identify the injector nozzle geometry.

The experimental measurements of fuels injection rates, signals of injector energizing, and total injected fuel mass were performed on the injection system test bench. The injection rate indicator IAV model EVI2 K-00-49 was used for the determination of experimental fuel injection rates. The signals of energizing times (ET) for the solenoid valve of the used injector were measured using an AC/DC current clamp Hantek ${ }^{\circledR}$ CC 65 . The total injected fuel mass was measured by a gravimetric balance KERN PFB 3000-2. A schematic diagram of the experimental setup can be seen in Figure 1.

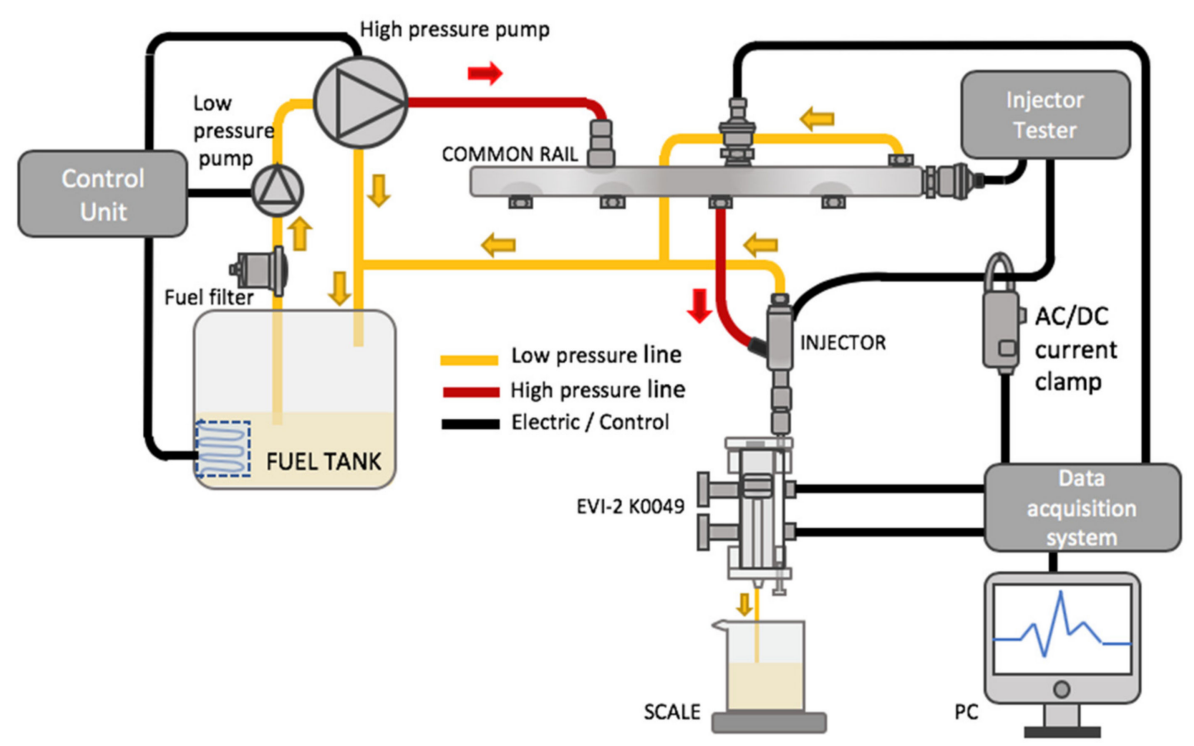

Figure 1. Schematic diagram of the experimental setup.

The operating engine mode selected to obtain the experimental data was $1700 \mathrm{rpm}$ of engine speed and $110 \mathrm{Nm}$ of load. The engine operating parameters used in the experimental set-up are presented in Table 2. When the engine runs at this operating mode, it produces two injections (one pre-injection and one main injection). However, in this study, only the main injection process has been considered. A detailed description of the experimental work and experimental set-up is presented in references [31,32]. 
Table 2. Operating parameters.

\begin{tabular}{|c|c|c|c|c|c|c|}
\hline \multirow[b]{2}{*}{ Fuel } & \multirow[b]{2}{*}{ Engine Speed (rpm) } & \multirow[b]{2}{*}{ Torque (Nm) } & \multirow{2}{*}{ Injection Pressure (bar) } & \multicolumn{2}{|c|}{$\mathrm{ET}(\mu \mathrm{s})$} & \multirow{2}{*}{$P_{\text {back }}$ (bar) } \\
\hline & & & & Pre-Injection & Main Injection & \\
\hline $\begin{array}{l}\text { Diesel } \\
\text { GTL }\end{array}$ & 1700 & 110 & $\begin{array}{l}845 \\
820\end{array}$ & 340 & 500 & 60 \\
\hline
\end{tabular}

\section{Numerical Models}

The influence of D2 and GTL fuel properties on the injection process was studied using the 1D AVL BOOST Hydsim computational program, where the whole injector, with the solenoid valve, was modeled. Numerical simulations of fuel flow and cavitation inception in the injection nozzle tip were performed using the 3D computational program AVL FIRE v2018. Table 3 shows the operating conditions used in numerical simulations.

Table 3. Operating modes used in numerical simulations.

\begin{tabular}{|c|c|c|c|c|c|}
\hline Model & Fuel & Engine Speed (rpm) & Torque (Nm) & Injection Pressure (bar) & $P_{\text {back }}$ (bar) \\
\hline $1 \mathrm{D}$ & $\begin{array}{c}\text { Diesel } \\
\text { GTL }\end{array}$ & 1700 & 110 & $\begin{array}{l}845 \\
820\end{array}$ & 60 \\
\hline $3 \mathrm{D}$ & $\begin{array}{c}\text { Diesel } \\
\text { GTL }\end{array}$ & 1700 & 110 & $\begin{array}{l}845 \\
820\end{array}$ & $40 / 60 / 80$ \\
\hline
\end{tabular}

\subsection{D Model}

The 1D model of the whole injector was divided into several parts, as presented in Figure 2. The first part (a) shows pressure input, where the injection pressure was specified for each fuel. The defined injection pressures were 845 bar for diesel fuel and 820 bar for GTL fuel, respectively. The difference in injection pressures results from the experimental set-up, where some parameters of the injection process were optimized for the need to operate the test engine at the desired speed of $1700 \mathrm{rpm}$ and the load of $110 \mathrm{Nm}$. The second part (b) is the injector body with all bores for fuel flow, needle, control piston, spring, injection orifice, and return line. The third part (c) presents a solenoid valve that contains inlet and outlet orifices, volumes, and a mechanical connection. The pulses for the opening of the solenoid valve, taken from experiments, were defined here. The in-cylinder pressure was set in the fourth part (d). The assigned value was $60 \mathrm{bar}$, which coincides with the experimental set-up. The last part (e) represents the fuel return line to the fuel tank.

The 1D Hydsim program was used to test the influence of fuel properties on the injection process, and to obtain the required needle lift data for each fuel, which was necessary for further simulations in the FIRE computational program. More information about the program and the models implemented in the program can be found in [33].

\subsection{D Model}

The 3D numerical model was made for one half of one injection hole, which is onefourteenth of the whole injection nozzle tip since it consists of seven injection holes. Figure 3 shows the computational mesh of the fuel injector and its 3D model. 


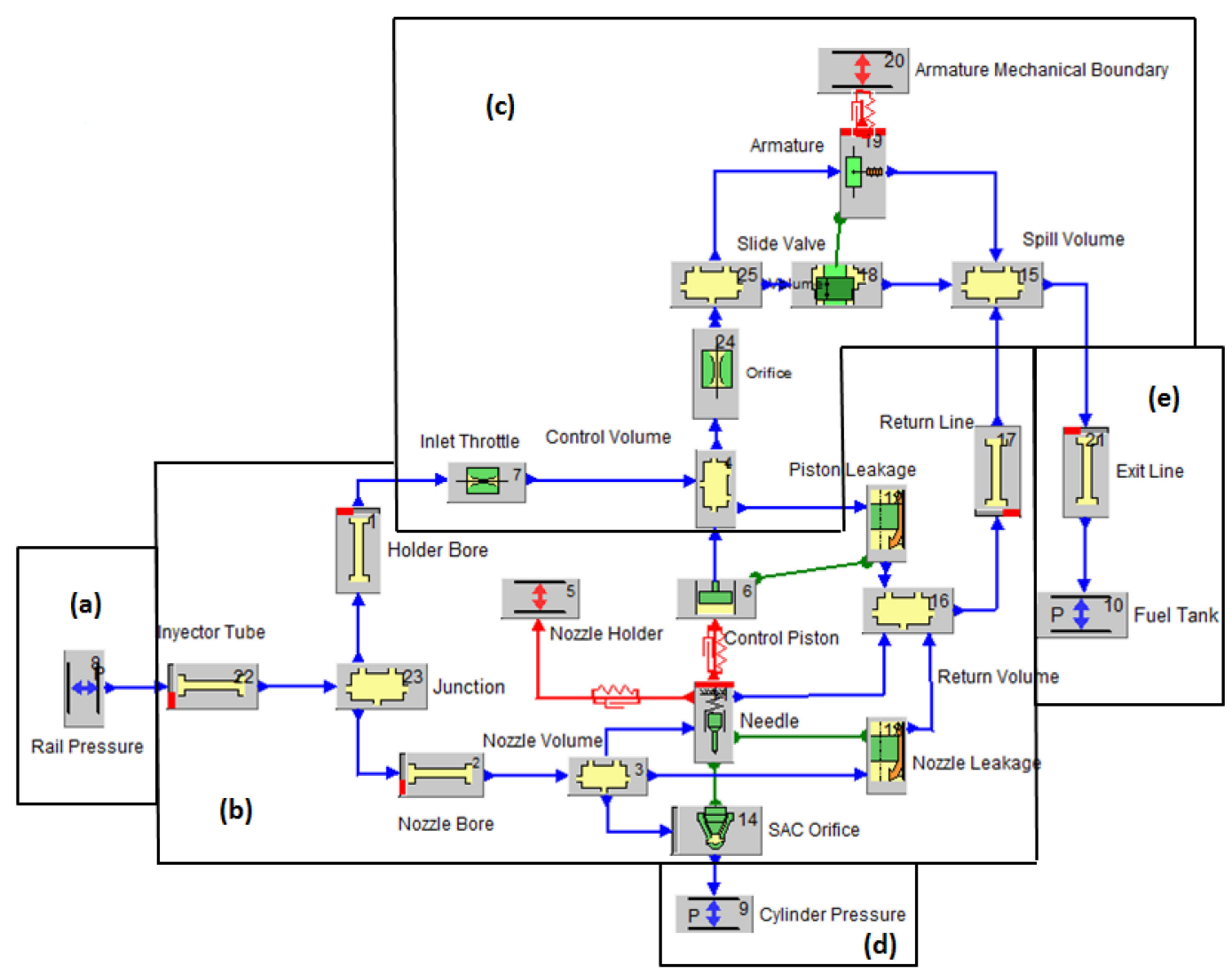

Figure 2. 1D Model of tested injector. (a) Rail Pressure; (b) Injector Body; (c) Solenoid Valve; (d) In-Cylinder Pressure; (e) Return line the fuel tank.

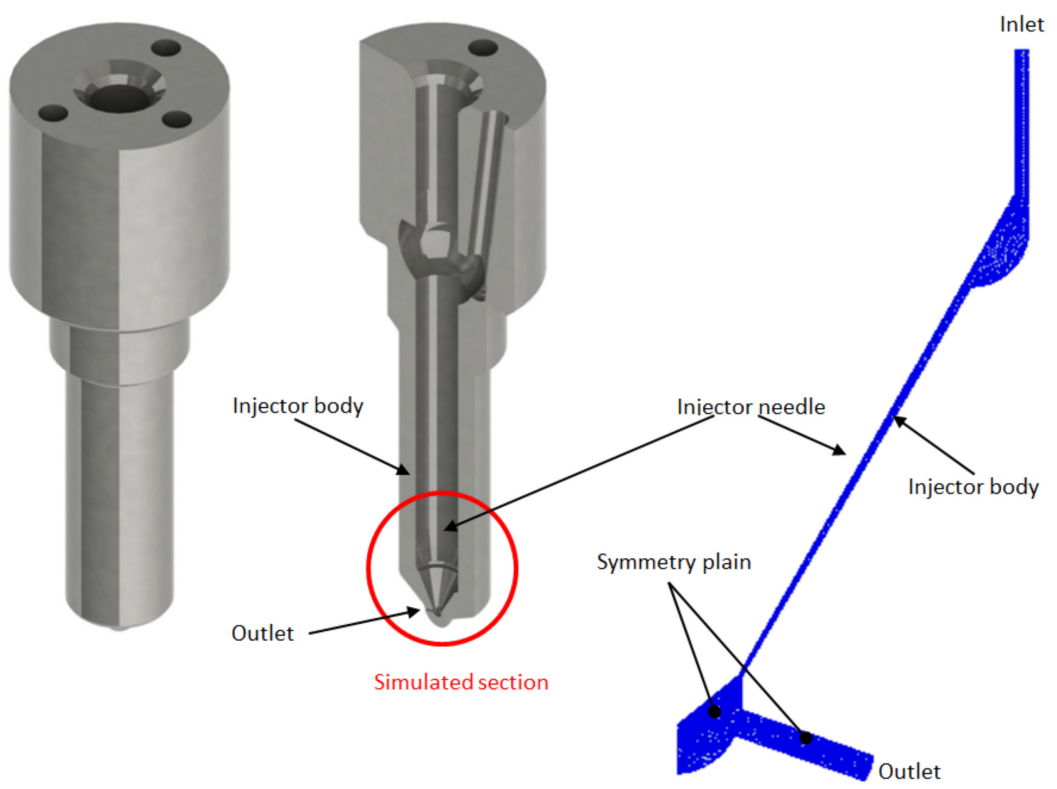

Figure 3. Injector muzzle model and computational mesh in the nozzle.

\subsubsection{Model Validation}

To perform the numerical simulation, the domain has to be discretized. Since the mesh density and time steps influence the results of transient numerical simulations, the mesh and time independence test were made for validating the numerical model. The 
independence test has also validated the suitability of the selected computational mesh and time steps for further studies. The mesh and time independence studies were made using diesel fuel properties, diesel fuel needle lift and 60 bar of in-cylinder back pressure.

\subsubsection{Mesh Independence}

The mesh independence test was carried out in order to select the optimal mesh and to ensure the independence of the obtained numerical results. Three different meshes with different mesh element densities were made and tested. The number of elements of each mesh is presented in Table 4 . When selecting values of time steps for the mesh independence test, we have followed the recommendation for time step values given in [23]. The results obtained using the three tested meshes are illustrated in Figure 4a.

Table 4. Approximate number of tested meshes elements for mesh independence study.

\begin{tabular}{cccc}
\hline Parameter & Mesh 1 & Mesh 2 & Mesh 3 \\
\hline Number of elements (millions of Hexcell elements) & 0.1 & 0.5 & 1 \\
\hline
\end{tabular}

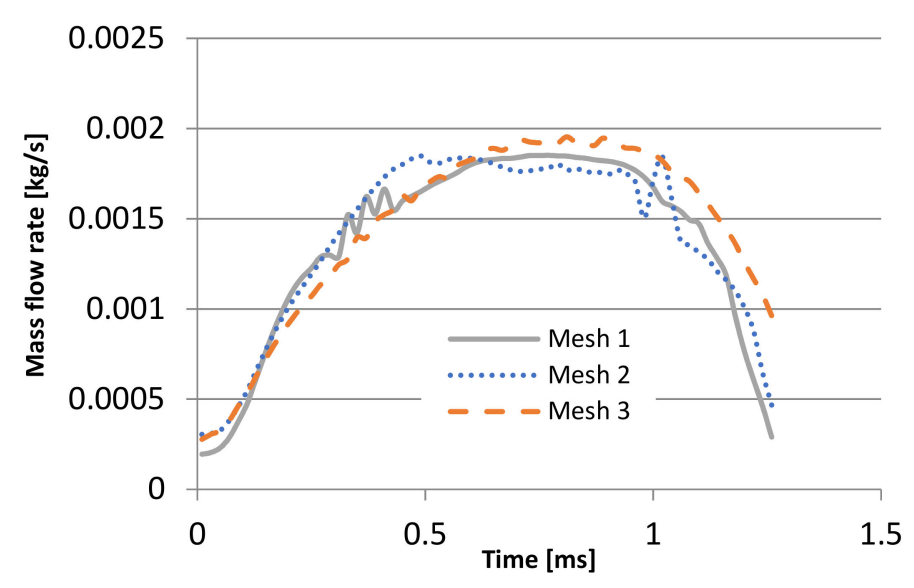

(a)

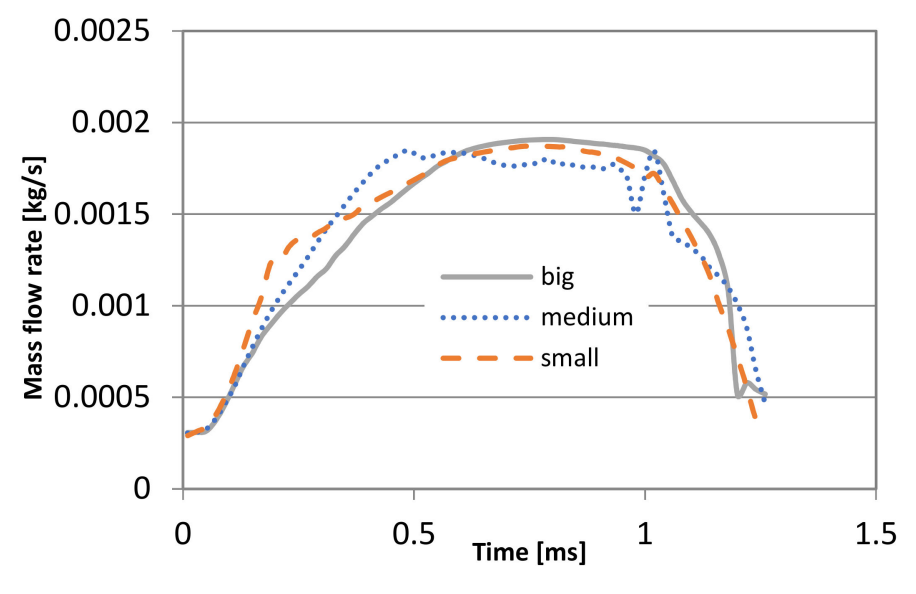

(b)

Figure 4. Results of the mesh independence study (a) and results of the time independence study (b): Evolution of mass flow rate over time.

The results obtained from the mesh independence test, Figure $4 \mathrm{a}$, indicate that all three tested meshes calculate a very similar fuel mass flow rate. When comparing the experimental and numerical results of total injected fuel mass calculated using different meshes, only small differences in results were obtained. The difference between injected fuel masses calculated using Mesh 1 and experimental results are 3\%, between Mesh 2, and experiment $1 \%$ and $2 \%$ between Mesh 3 and experimental results. The medium-sized mesh, Mesh 2, with 0.53 million of Hexcell elements, was the selected mesh for further work.

\subsubsection{Time Independence}

The time independence study was made only for the selected computational mesh (Mesh 2). The chosen time steps were ten times smaller and ten times larger than in the case with medium time steps. The time steps for the medium case were selected according to the recommendation provided in [23]. Table 5 shows all tested time steps. The start time steps were smaller than at the end in order to obtain good convergence. 
Table 5. Time steps for time independence study.

\begin{tabular}{cccc}
\hline Time (s) & Big & Medium & Small \\
\hline Start & $2.5 \times 10^{-7}$ & $2.5 \times 10^{-8}$ & $2.5 \times 10^{-9}$ \\
\hline End & $5 \times 10^{-6}$ & $5 \times 10^{-7}$ & $5 \times 10^{-8}$ \\
\hline
\end{tabular}

The diagram presented in Figure $4 \mathrm{~b}$ shows the results of the time independence study for different time steps. From the results, a slight influence of time step on fuel mass flow rate at nozzle exit can be observed. When comparing the numerical results of total injected fuel mass per cycle with experimental results we obtained only a small difference in total injected fuel mass. The difference when using big time step is $1.6 \%, 1.1 \%$ when using medium time steps, and $0.8 \%$ when using small time steps. According to the presented comparison, medium time steps give results with good accuracy in reasonable computational times; therefore, they were selected for further work.

\subsubsection{Simulation Set-Up}

The physical total time of simulations was equal to the total times of injection. For diesel fuel, it was set to $1.26 \mathrm{~ms}$ and for GTL fuel to $1.16 \mathrm{~ms}$. The time steps of simulations ranged from $2.5 \times 10^{-8} \mathrm{~s}$ to $5.0 \times 10^{-7} \mathrm{~s}$ and were selected according to the previously presented time independence study. The smaller time steps were used at the start of simulations to obtain good convergence. At the inlet and outlet boundary, pressure values were specified. The inlet pressure values correspond to the injection pressures presented in Table 3, which also provides the values of back pressure $\left(\mathrm{p}_{\mathrm{back}}\right)$ used. The symmetry boundary condition was used on the two sides of the injector mesh. The properties of the used fuels, presented in Table 1, were implemented in the AVL FIRE program.

The transient needle movement was considered during the simulations. The needle's position during the injection process was determined from the results obtained with the AVL BOOST Hydsim program at 60 bar of in-cylinder back pressure. At the beginning of the simulations, the needle was not fully closed. This allowed us to avoid zero thickness cells and define the computational mesh in the area between the needle and the injector body. The minimal distance between the needle and the injector body was $20 \mu \mathrm{m}$. A similar approach was proven to give good results in $[23,34]$.

In order to reduce the time of numerical simulation, the start of needle lift was used as the start of simulations in the AVL FIRE program. The curves of needle lift for each fuel were the same for all selected values of in-cylinder back pressure. This allowed us to study only the influence of back pressure on the conditions inside the injection nozzle without additional variables.

\subsection{Mathematical Models}

Three different approaches are commonly used when simulating cavitation flow within nozzle holes. These approaches use the homogeneous flow approach, the volumeof-fluid approach, or the two-fluid approach. The two-fluid model approach calculates all conservation equations for each phase, while the other two approaches calculate the volume fraction of each phase and solves only single momentum or enthalpy equations [35]. As shown in [36], the two-fluid model approach gives a detailed description of the flow in less computational time. According to this, the two-fluid model, integrated into the used FIRE software, was used in the presented study. Some of the important models' equations are presented in this paper. A detailed description of the models can be found in [35].

The energy equation was used to include the influence of density changes on the flow field. The governing mass, momentum, and energy conservation equations, based on Reynolds averaged Navier-Stokes (RANS) equation, can be written as follows:

$$
\frac{\partial \alpha_{k} \rho_{k}}{\partial t}+\nabla \cdot \alpha_{k} \rho_{k} v_{k}=\sum_{l=1, l \neq k}^{2} \Gamma_{k l}
$$




$$
\begin{aligned}
\frac{\partial \alpha_{k} \rho_{k} v_{k}}{\partial t}+ & \nabla \cdot \alpha_{k} \rho_{k} v_{k} v_{k}=-\alpha_{k} \nabla p+\nabla \cdot \alpha_{k}\left(\tau_{k}+\tau_{k}^{t}\right)+\alpha_{k} \rho_{k} g+v_{k} \sum_{l=1, l \neq k}^{2} \Gamma_{k l}+\sum_{l=1, l \neq k}^{2} \mathrm{M}_{k l} \\
\frac{\partial \alpha_{k} \rho_{k} h_{k}}{\partial t}+\nabla \begin{aligned}
{ }^{\prime} \\
\quad
\end{aligned} & \alpha_{k} \rho_{k} v_{k} h_{k} \\
& =\nabla \cdot \alpha_{k}\left(q_{k}+q_{k}^{t}\right)+\alpha_{k} \rho_{k} q_{k}^{\prime \prime \prime}+\alpha_{k} \rho_{k} f \cdot v_{k}+\nabla \cdot \alpha_{k}\left(\tau_{k}+\tau_{k}^{t}\right) \cdot v_{k}+\alpha_{k} \frac{\vartheta p}{\vartheta t}+\sum_{l=1, l \neq k}^{2} \mathrm{H}_{k l} \\
& +\sum_{l=1, l \neq k}^{2} \mathrm{~h}_{k i} \Gamma_{k l}
\end{aligned}
$$

where k equals 1 for the pure gas phase and 2 for the pure liquid phase only. $v_{k}$ and $\rho_{k}$ are the velocity and density at phase $k . \Gamma_{k l}$ denotes the interfacial mass transfer between phases $k$ and $l, \tau_{k}$ represents the shear stress, and $\tau_{k}^{t}$ the Reynolds stress at phase $k . \mathrm{M}_{k l}$ is the interfacial momentum transfer and $\mathrm{H}_{k l}$ is the interfacial energy transfer between phases $k$ and $l . \alpha_{k}$ is the volume fraction of phase $k . q_{k}$ is the heat flux, $q_{k}^{t}$ is the turbulent heat flux, and $q_{k}^{\prime \prime \prime} 0$ the enthalpy volumetric source. The sum of volume fractions is calculated using Equation (4) as:

$$
\sum_{k=1}^{2} \alpha_{k}=1,
$$

The Tait equation (Equation (5)) was used to calculate the changes in fuel density at high pressure, where $\rho_{r e f}$ and $p_{r e f}$ denote the reference density and pressure. $\mathrm{B}$ and $\mathrm{n}$ are empirical constants.

$$
\rho_{l}=\rho_{r e f} \sqrt[n]{\frac{p+\mathrm{B}}{p_{r e f}+\mathrm{B}}}
$$

The interfacial mass exchange was calculated using linear cavitation as follows in Equation (6), where $N^{\prime \prime \prime}$ is the bubble number density, $\mathrm{R}$ is the mean bubble radius in the cavitation region and $\dot{R}$ is the change rate of bubble radius. The linear cavitation model predicts that the governing force for mass exchange is the pressure difference between phases.

$$
\Gamma_{12}=\rho_{d} N^{\prime \prime \prime} 4 \pi R^{2} \dot{R}=\Gamma_{21}
$$

In the present work, the turbulence modeling was done using a standard k- $\varepsilon$ turbulence model. The model was selected based on works presented in $[21,25,29,34]$. Hybrid wall treatment, suitable for all $\mathrm{y}+$ values, was used to compute the near wall region's turbulent flow.

Additional interfacial momentum exchange was considered in the simulations. The model was used to take into account the effect of drag and turbulence dispersion, while inertia and lift effects were neglected. More about the used model can be found in [35].

\section{Discussion}

The influence of the physical-chemical properties of the tested fuels and in-cylinder back pressure on the injection process, in-nozzle flow, and cavitation inception was tested in the presented paper. The study was made numerically, using a modern injector with a solenoid valve taken from the common rail injection system of a Nissan diesel engine. First, the influence on the injection process was tested numerically, using the one-dimensional program, and then experimentally. The obtained results were used further for three-dimensional simulations, where we used only one-fourteenth of the whole injection nozzle tip and considered transient needle motion during the injection process. In the following chapters, only the most relevant results are presented at different times of the injection process.

\subsection{Influence on Injection Process}

In the presented study, only the main fuel injection, without pre-injection, was modeled. The results of numerical simulations (NS) were compared to the experimentally 
obtained results (EXP). The results of fuel mass flow rates and needle lifts are presented in Figure 5.

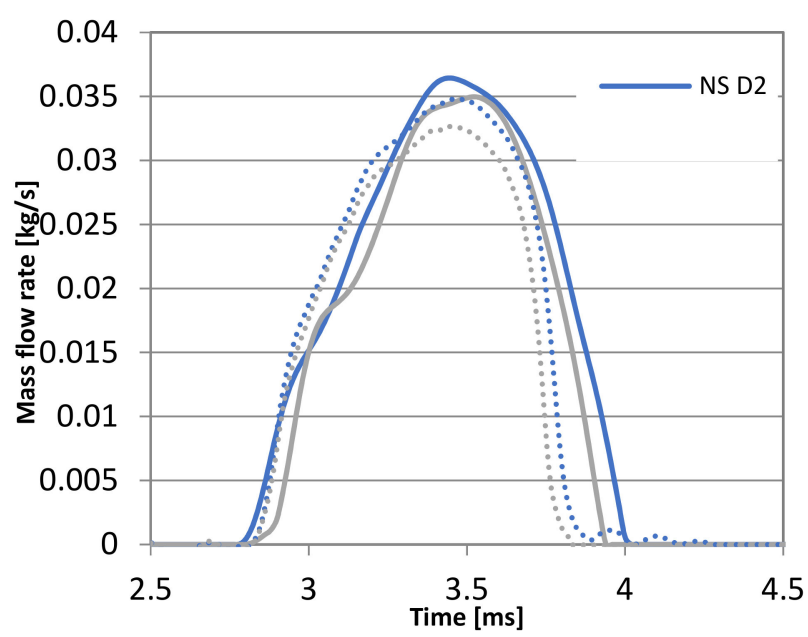

(a)

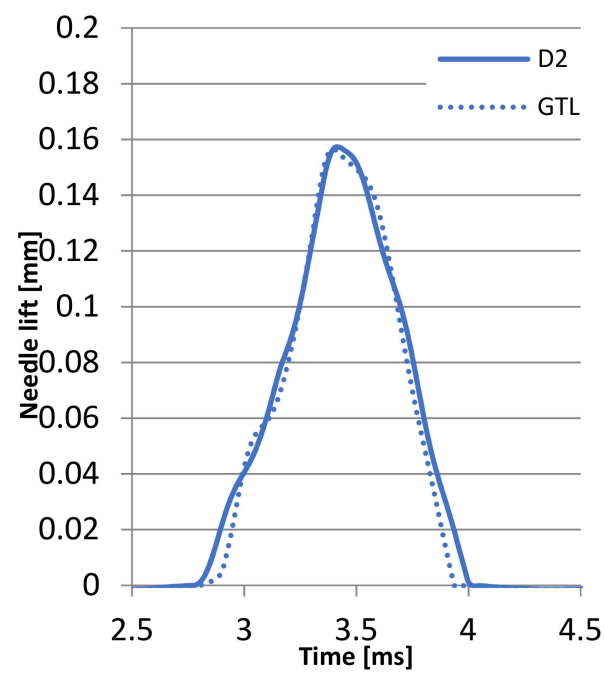

(b)

Figure 5. Influence on injection process: (a) Experimentally and numerically obtained fuels mass flows for a whole injector: Evolution of mass flow rate over time; (b) Numerically obtained needle lift for D2 and GTL fuels.

The results regarding fuel mass flow, Figure 5a, show that the Hydsim program predicts slightly higher maximal values of fuel mass flows for diesel (NS D2) and GTL (NS GTL) fuels. Hydsim also over predicts the total time of injection slightly, so, by the end of the injection process, we have higher values of fuel mass flow compared to the experiment.

The injection duration of diesel fuel is longer compared to GTL fuel. The reason for this is the longer energizing time of the solenoid valve when diesel fuel is used. These experimental results were obtained from a Nissan engine, where injection duration was optimized in order to operate the test engine at the desired engine speed and load. The numerically calculated start of injection was at $2.78 \mathrm{~ms}$ for diesel fuel and $2.8 \mathrm{~ms}$ for GTL fuel. In the experiment, the injection of both GTL and D2 fuel started at $2.82 \mathrm{~ms}$. The duration of the numerically obtained injection process for D2 and GTL fuels is $1.26 \mathrm{~ms}$ and $1.16 \mathrm{~ms}$, respectively. The total injection duration during the experiment was $1.07 \mathrm{~ms}$ for D2 and $1.01 \mathrm{~ms}$ for GTL fuel.

In order to consider dynamic needle motion in the 3D simulation, we require needle lift in each time of the injection process. The obtained needle lifts for diesel and GTL fuels are presented in Figure 5b. A clear connection between needle lift and mass flow rate can be seen from the results. A start of needle lift for D2 fuel is a little advanced compared to GTL fuel, which influences on advanced diesel fuel injection (mass flow). The gradient of the GTL fuel needle lift is steeper shortly after the injection start, which leads to higher needle lift for GTL fuel at around $3 \mathrm{~ms}$ and causes higher GTL fuel mass flow at the same time of injection.

The time at needle lift start for each fuel was taken as the initial time for 3D simulations. As there is no injection process or internal fuel flow before this time, there is no point in simulating when the needle is closed.

The higher value of diesel fuel density, compared to GTL fuel, slightly increases its fuel mass flow obtained numerically and experimentally. This fact affects the total injected fuel mass per injection cycle further, as presented in Figure 6. 


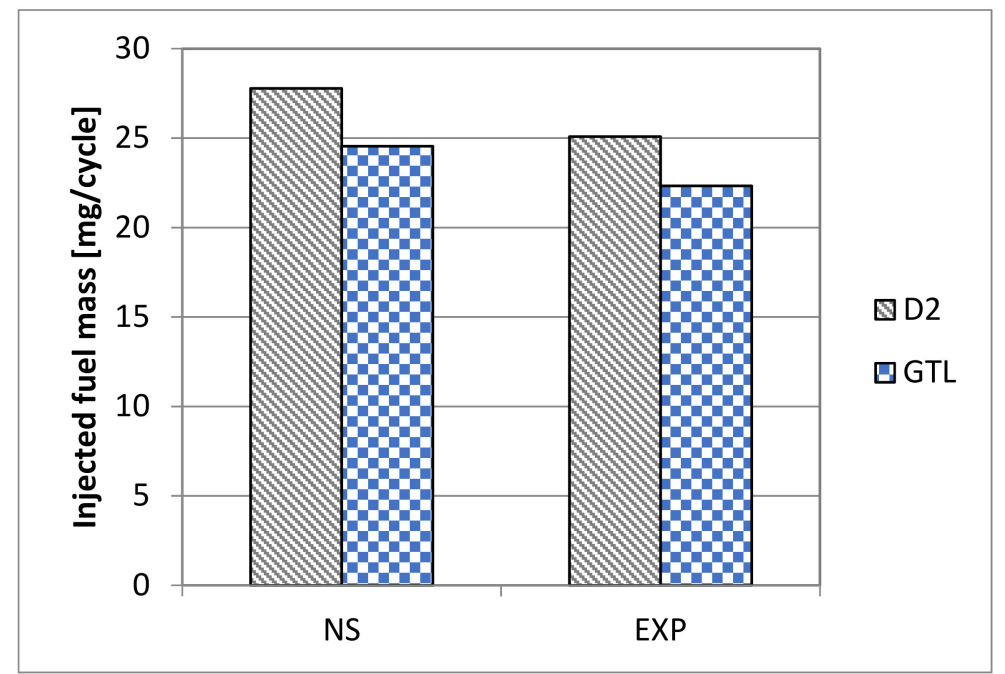

Figure 6. Numerically and experimentally obtained results of total injected fuel mass per cycle.

The numerical and experimental results of injected fuel mass presented in Figure 6 show higher injected mass for diesel fuel. This is a result of the higher maximal values of diesel fuel mass flow due to higher diesel fuel density. Numerically obtained values of injected fuel masses are approximately $10 \%$ higher than experimental values. This is a consequence of a longer injection duration predicted by the numerical Hydsim program.

The comparison of fuel mass flows and total injected fuel masses show good agreement between numerical and experimental results, which indicate that results from the BOOST Hydsim program (needle lift) can be used for further research.

\subsection{In-Nozzle Flow and Cavitation Inception}

In the following sections, only the most relevant results are presented at different times of the injection process. The results are divided into two sub-sections. First, the influence of fuel properties on flow characteristics is discussed at 60 bar of in-cylinder back pressure. The influence of in-cylinder back pressure was tested in the following step.

\subsubsection{Influence of Fuel Properties}

The influence of fuel properties on fuel flow condition and cavitation inception inside the injector was studied numerically. When fuel flows through the injector it undergoes significant changes in flow direction, which causes fluctuation in velocity and pressure. This further influences the formation of cavitation. There are two regions inside the injector where most of the cavitation structures are formatted. The fuel undergoes the most significant changes in flow direction when it flows into the injection hole. This change in flow direction causes the formation of an area with very low pressure at injection hole entry, marked in Figure 7. Because the pressure at the nozzle exit is usually lower than the fuel pressure inside the injector SAC volume and inside the injection hole, the formed cavitation structures travel with the fuel flow and stretch throughout the injection hole towards the nozzle exit denoted with S1 at Figure 7. The second area of cavitation formation is in the space between the injector needle and the injector body, which is very narrow when opening and closing the needle. Since the most significant area of cavitation formation is at the fuel entry to the injection hole, the second area was not studied in the scope of the work presented in this paper. The schematic presentation of the injection hole entry and different locations inside the injection hole are presented in Figure 7. 


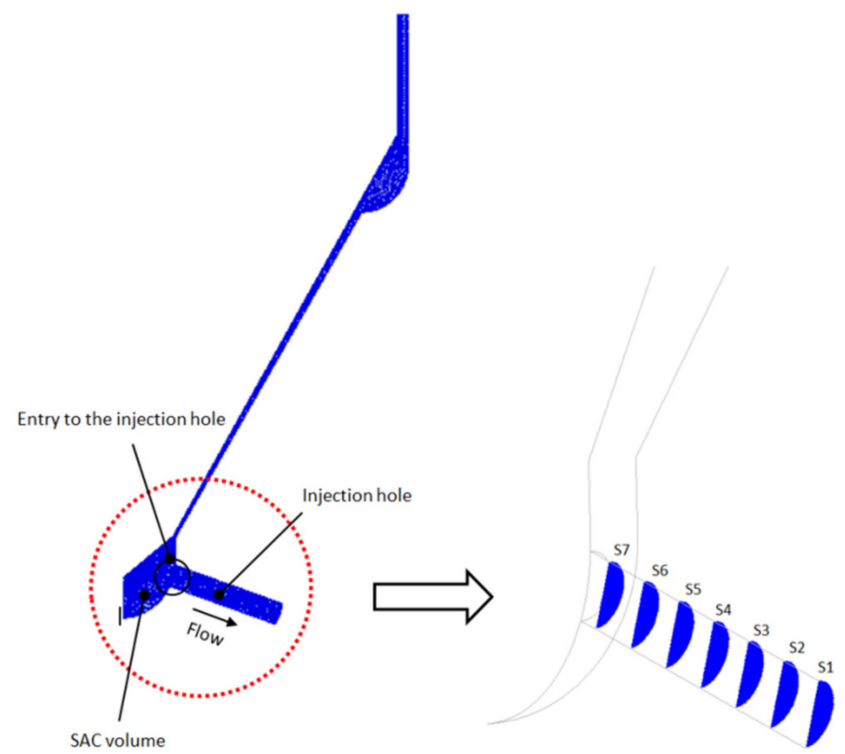

Figure 7. Schematic presentation of injection hole, nozzle SAC volume and location (slices) for presentation of results inside the injection hole.

The results of the vapor volume fraction at different locations (S1 to S7) inside the injection hole are presented in Figure 8. The results for D2 and GTL fuels are provided at the same time during the injection process. The results in Figure $8 \mathrm{f}$ show the last simulation time step, which differs in time for each tested fuel.

The results indicate that both fuels induce cavitation formation (denoted with a light blue, green, yellow, and red color) in the upper part of the injection hole entry. Since the injector has seven injection holes, the majority of the fuel which flows under the injector needle enters directly to the injection holes, and not to the nozzle SAC volume, marked in Figure 7 . This creates the area of low pressure at the upper part of the injection hole entry, which further induces the cavitation formation [24]. Since both fuels undergo significant changes in flow direction and the pressure drop is very high (practically to $0 \mathrm{~Pa}$ of absolute pressure [23]), the differences in the cavitation area for both fuels are quite small. Slightly wider regions of the cavitation area were obtained using diesel fuel. This can be related to higher values of diesel fuel saturation pressure. The difference in cavitation area can also be related to difference in fuel kinematic viscosity. The higher viscosity of GTL fuel results in less cavitation. This agrees with the results of Saleh at al. [37], and Nouri et al. in [38] where they also concluded that higher viscosity of fluid results in less formatted cavitation. At needle closing, cavitation vapor fraction dissipates (declines) later, so it was still visible on S6 and S7 at $1 \mathrm{~ms}$ after the injection starts, when the needle is almost closed.

Figure 9 presents the results of normalized maximal values of Vapor Volume Fraction for diesel and GTL fuel at slices S1 to S7 of the injection hole. From these results, the movement of cavitation structures through the injection nozzle can be observed. As can be seen, the cavitation formation is first triggered at fuel entry to the injection hole (S7). For diesel fuel, this happened at $0.26 \mathrm{~ms}$ after the injection start, while for GTL fuel at $0.29 \mathrm{~ms}$. This coincides with the results from Figure 10a where no Vapor Volume Fraction (VVF) is visible at $0.25 \mathrm{~ms}$ after the injection start. In Figure $8 \mathrm{~b}, 0.5 \mathrm{~ms}$ after the injection start, the VVF can be noticed for both fuels. Since diesel fuel advances the cavitation formation, the VVF can be noticed on the slices from S7 to S1, while for GTL fuel, it is only from S7 to S5 at the time $0.5 \mathrm{~ms}$ after the injection start. The fluctuation of VVF inside the injection nozzle can be seen around $0.8 \mathrm{~ms}$ for D2 fuel and at $0.7 \mathrm{~ms}$ for GTL fuel. This can be noticed as a decrease in the normalized VVF values, Figure 9. The fluctuation of the cavitation structure is more evident for diesel fuel, where a drop in VVF was obtained at all slices of the injection hole. 


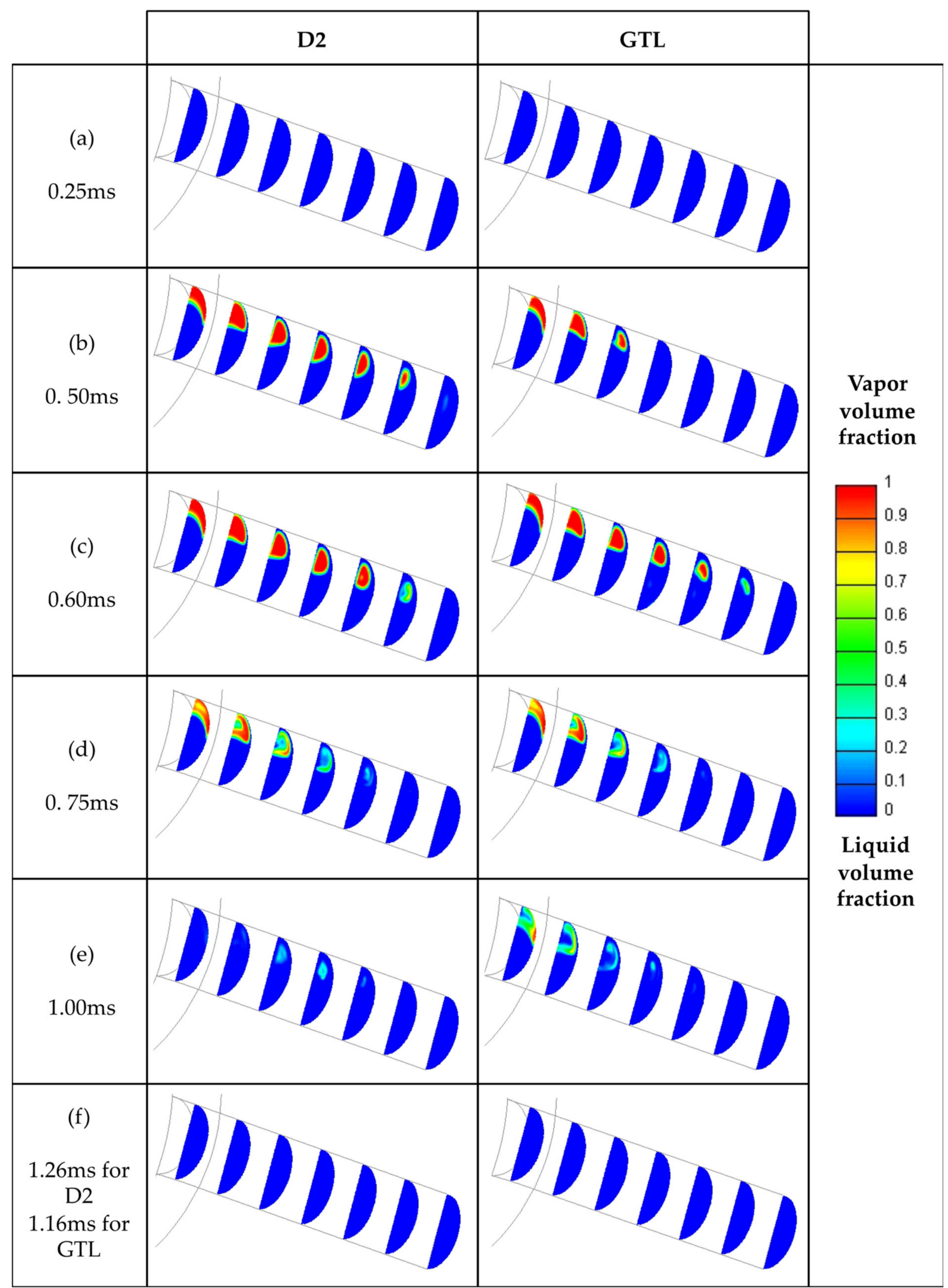

Figure 8. Vapor Volume Fraction at 60 bar of in-cylinder pressure. 
(a)

(b) (c)

(d)

(e)

(f)

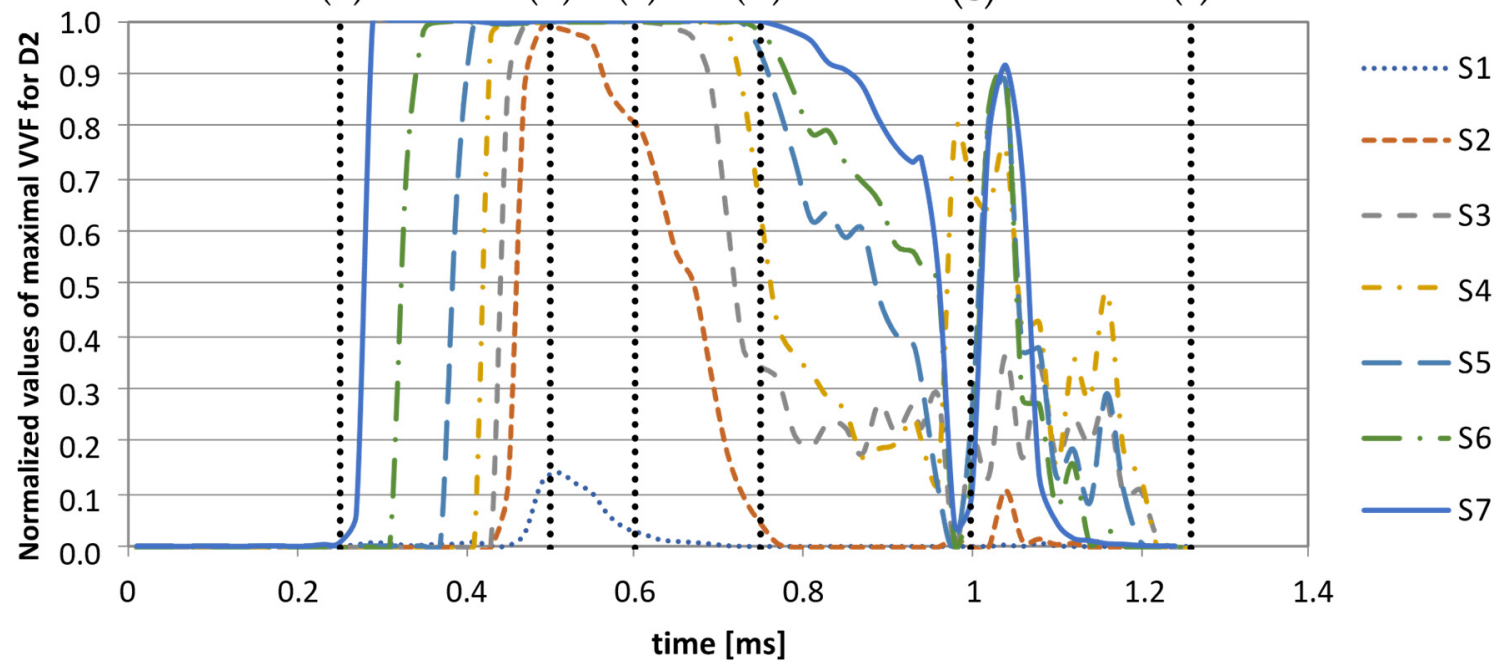

(a)

(a)

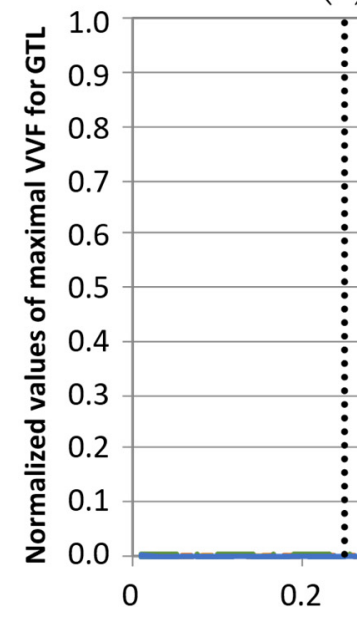

(b) (c)

(d) (e)

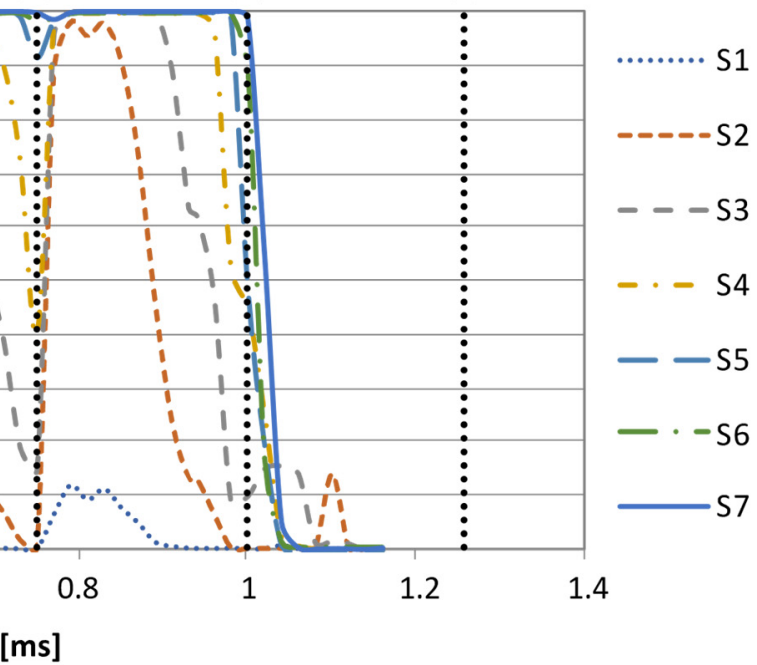

(b)

Figure 9. Volume fraction curved diagram at 60 bar of in-cylinder pressure, (a) with D2; (b) with GTL.

The results for fuel mass flow, average normal velocity, average turbulent kinetic energy, and maximal values of vapor volume fraction at the nozzle exit are presented in Figure 10.

The results of the fuel mass flow rate show that the mass flow for D2 fuel starts to rise earlier than in the case of GTL fuel, Figure 10a. This leads to higher fuel velocity and faster formation of cavitation structures at the start of the injection process. There is a slight increase in the mass flow of GTL at $0.8 \mathrm{~ms}$. This further influences cavitation formation, which starts to decline later in the injection process compared to D2 fuel, Figure 8. The fluctuation in fuel mass flow also influences the average normal fuel velocity at the nozzle hole exit, see Figure 10b. During most of the injection process, diesel fuel showed slightly higher velocities and mass flow rates than GTL. This can be related to its lower kinematic viscosity compared to GTL fuel $[23,28-30]$. The same was also found in work of Battistoni et al. [23], where they concluded that lower fuel viscosity results in lower pressure losses inside the injection hole. 


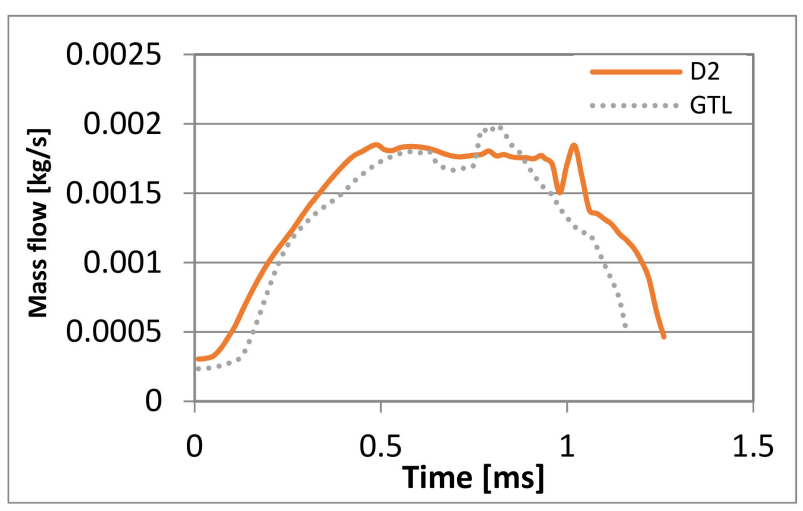

(a)

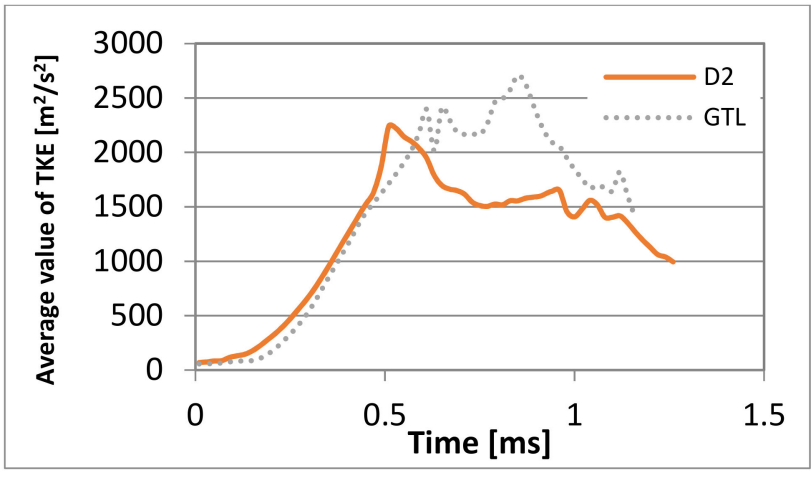

(c)

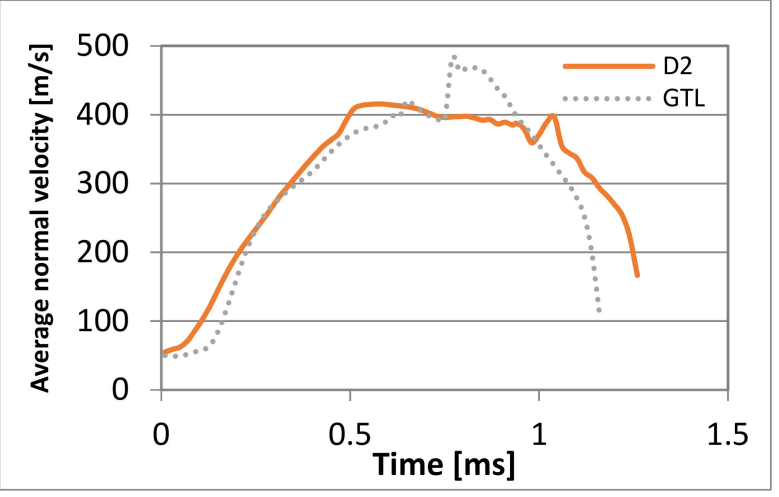

(b)

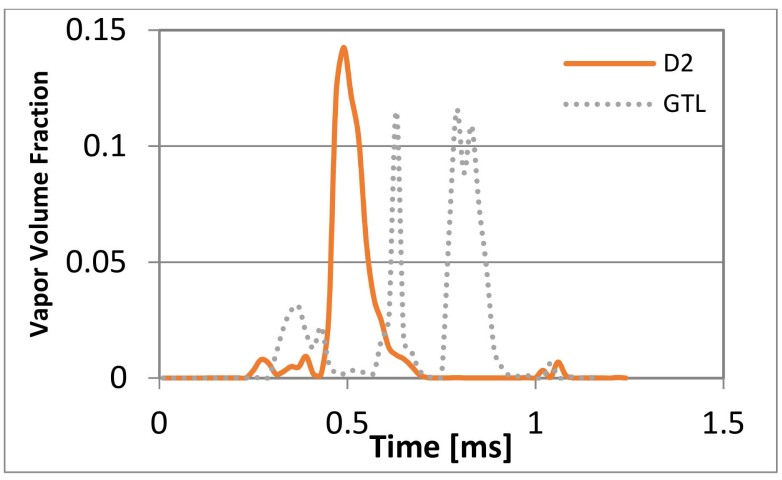

(d)

Figure 10. Outlet integral quantities for $\frac{1}{2}$ of the injection hole: (a) Mass flow rate; (b) Average normal velocity; (c) Average value of liquid turbulent kinetic energy; (d) Maximal value of Vapor Volume Fraction.

Figure 10c presents the results of the average values of turbulent kinetic energy (TKE) at the nozzle exit hole. The value of the average TKE increases with the increase in mass flow. The maximal values of average TKE at the nozzle exit hole collide with the time when the maximal values of mass flow, normal fuel velocity, and Vapor Volume Fraction appear at the nozzle outlet (see Figure 10d). This indicates that the magnitude of turbulent kinetic energy increases with the increase of the fuel mass flow rate, but it is also influenced by the appearance of the vapor phase at the nozzle exit hole.

The results of the maximal values of VVF show three distinctive sections. At first, VVF appears at the nozzle outlet at the time of mass flow rise. In this first section, the values of VVF for GTL fuel are significantly higher than the values for diesel fuel. The second section indicates the Vapor Volume Fraction peaks for D2 and GTL fuels, which collide with the first peak of fuel mass flows. This occurs around $0.5 \mathrm{~ms}$ for D2 fuel and $0.6 \mathrm{~ms}$ for GTL fuel. In the second section, maximal values of VVF are obtained when using diesel fuel. The third section indicates the region where the maximal values of mass flows occur. For GTL fuel, this happens around $0.8 \mathrm{~ms}$ after the injection start, while for D2, shortly after $1 \mathrm{~ms}$ after the injection start. The first and third sections are distinctly more evident for GTL fuel, while for D2 fuel, only a slight bump in the VVF maximal values is noticed, which is hard to connect directly with the first peak, or with a maximal value of D2 mass flow rate.

\subsubsection{Influence of In-Cylinder Back Pressure}

The difference between common-rail fuel pressure and in-cylinder pressure is the governing force for the fuel flow in the injector nozzle. If these conditions change, which can happen in the case of a pre- or post-injection, they can influence fuel flow conditions, which further influences the cavitation formation. Two additional in-cylinder pressures of 40 and 80 bars were tested in the following section. 
Figures 11 and 12 show the result of cavitation vapor structure formation on different locations inside the nozzle at 40 and 80 bar of in-cylinder atmospheric pressure.

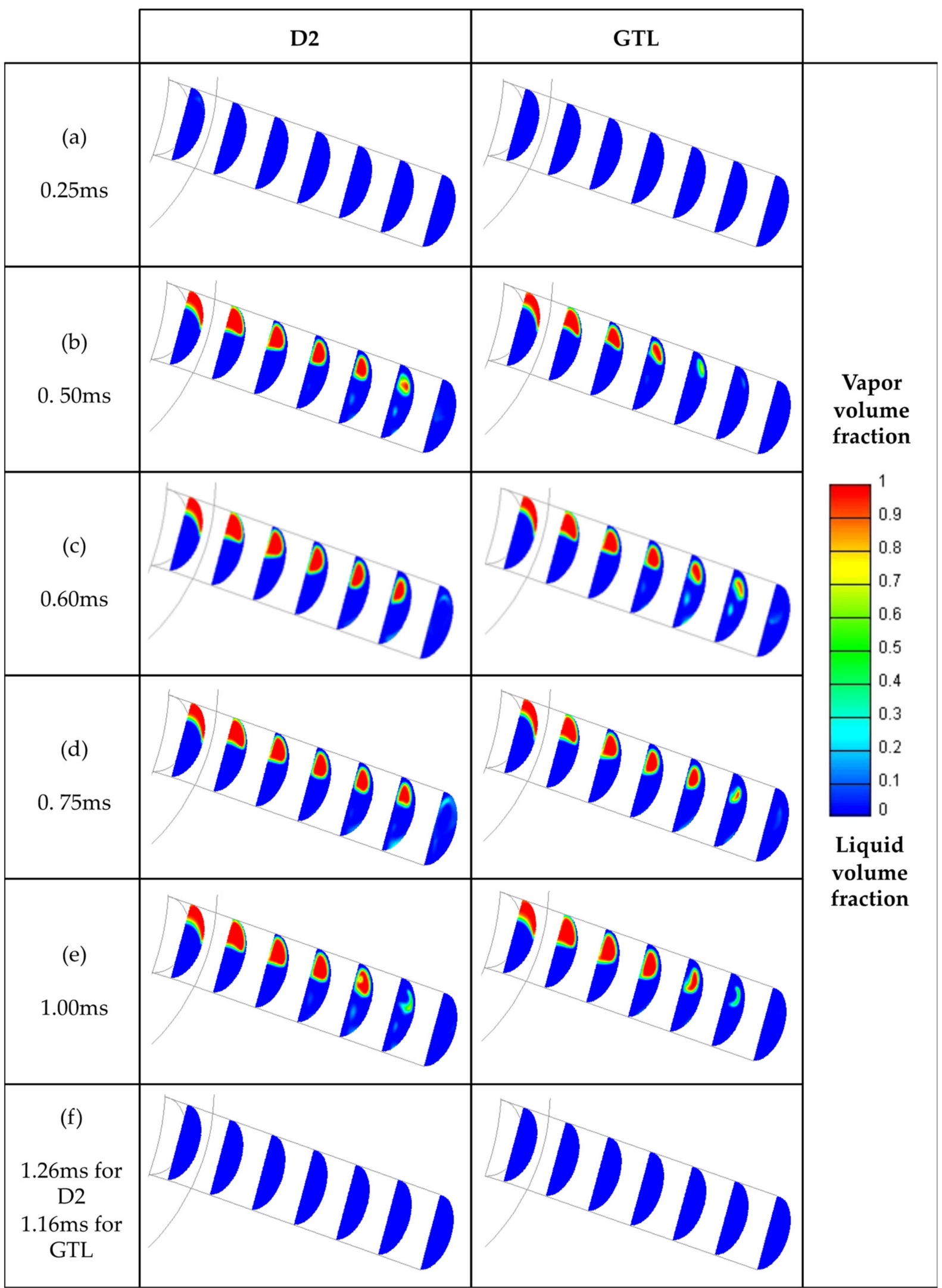

Figure 11. Vapor Volume Fraction at 40 bar of in-cylinder pressure. 


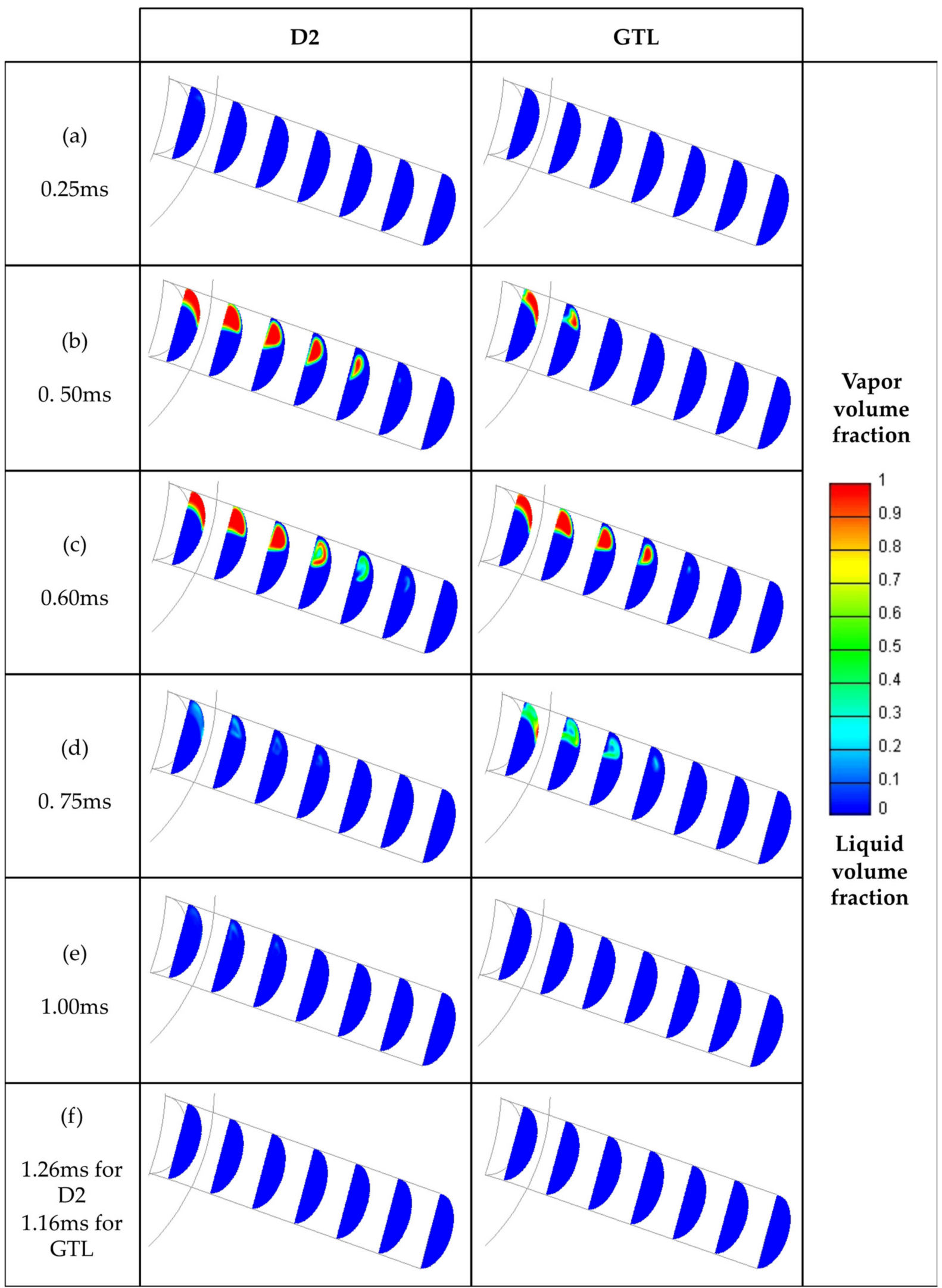

Figure 12. Vapor Volume Fraction at 80 bar of in-cylinder pressure.

The significant influence of in-cylinder back pressure on cavitation Vapor Volume Fraction formation can be seen in Figures 11 and 12. These results were expected since the pressure difference between injected fuel and in-cylinder pressure governs the injector's 
fuel flow. Similar trends were also observed by Dai et al. in [24], where a higher difference between fuel injection pressure and atmospheric pressure results in an increase in cavitation. The results for both fuels and both in-cylinder pressures indicate that cavitation appears at the upper part of the injection hole entry and then spreads towards the outlet, as in the case of 60 bar of in-cylinder pressure. The area of cavitation fraction at $0.5 \mathrm{~ms}$ is significantly higher when lower in-cylinder pressure is applied. A similar trend can be observed at needle closing (Figures 11e and 12e). At 40 bars of in-cylinder pressure, the cavitation is still fully developed at $1 \mathrm{~ms}$ after the injection start. In comparison, at 80 bars, the area of cavitation at this time of injection is negligible. As far as the fuel type influence is concerned, diesel fuel gives a slightly wider region of cavitation area compared to GTL fuel. The opposite trend is only evident at $80 \mathrm{bar}$ of in-cylinder pressure at $0.75 \mathrm{~ms}$, where GTL fuel cavitation vapor fraction dissipation (decline) is delayed, so there the cavitation area is wider (S4-S7, Figures 11 and 12). Wider regions of cavitation structures for diesel fuel are the result of the higher diesel fuel saturation pressure.

The results for fuel mass flow, average normal velocity, average turbulent kinetic energy, and maximal values of Vapor Volume Fraction at nozzle exit for different in-cylinder pressures are presented in Figure 13.

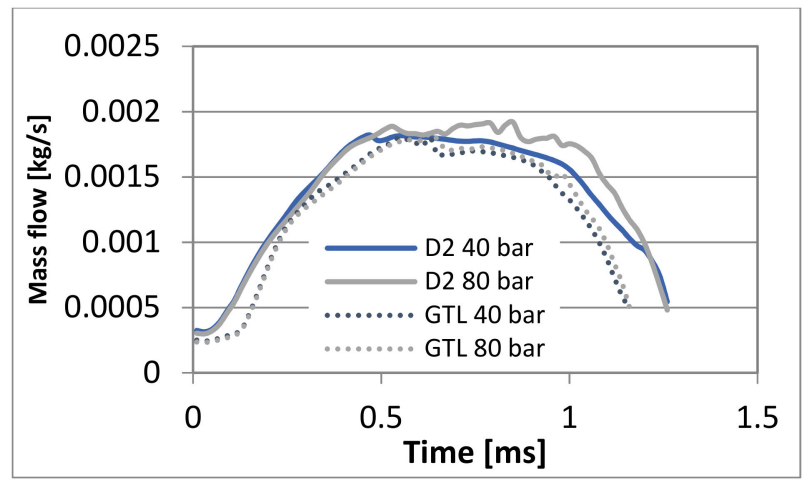

(a)

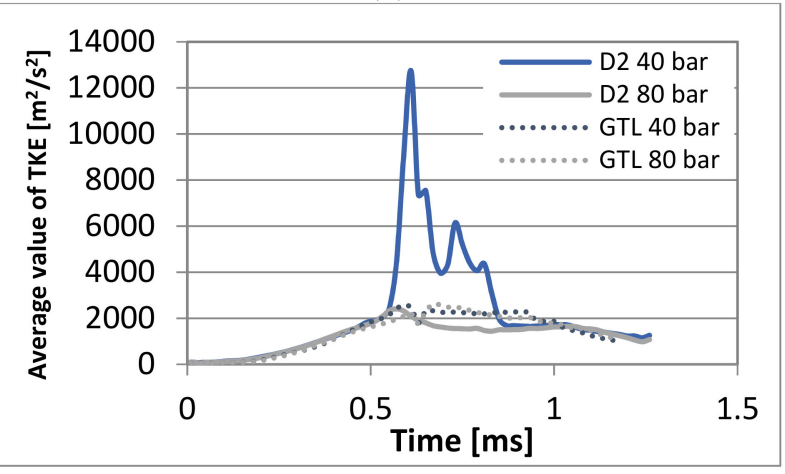

(c)

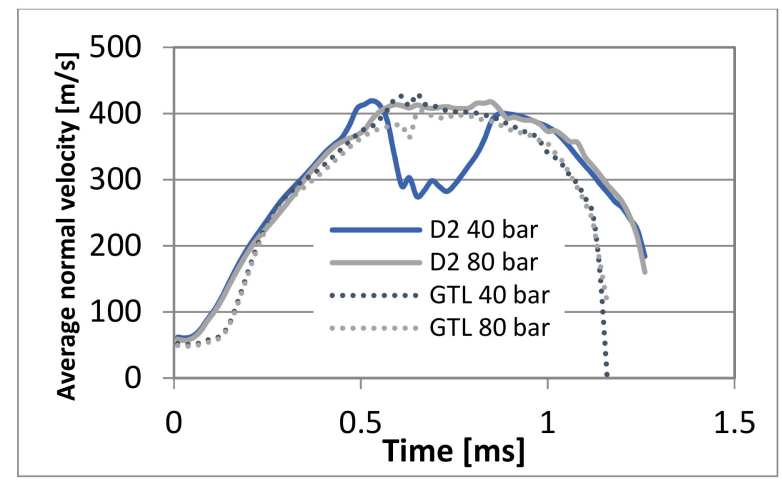

(b)

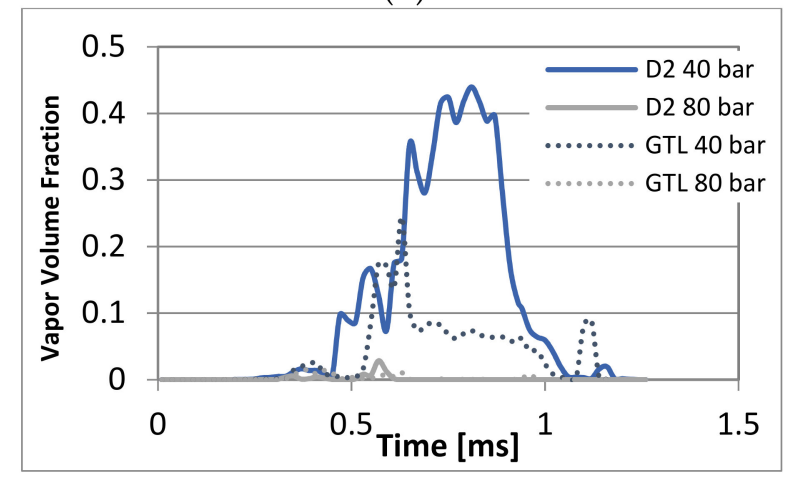

(d)

Figure 13. Outlet integral quantities for $\frac{1}{2}$ of the injection hole: (a) Mass flow rate; (b) Average normal velocity; (c) Average value of liquid turbulent kinetic energy; (d) Maximal value of Vapor Volume Fraction.

The results of fuel mass flow (see Figure 13a) indicate a slightly higher fuel mass flow for diesel fuel, which is a result of the higher diesel fuel density and lower kinematic viscosity. The fuel mass flow for diesel starts to increase earlier compared to GTL fuel at both in-cylinder pressures. Because of the shorter injection duration, the fuel mass flow for GTL starts to decline faster than for D2 fuel.

The fluctuation of fuel average normal velocity, average values of turbulent kinetic energy, and maximal values of Vapor Volume Fraction at the nozzle hole outlet is again strongly influenced by the value of fuel mass flow. The higher mass flow rates and higher velocities for D2 fuel can be attributed to the lower viscosity and higher density of diesel 
fuel. The lower viscosity of diesel fuel increases fuel velocities, which, combined with the higher diesel fuel density, increases the fuel mass flow rate.

The maximal fluctuations of fuel velocity, turbulent kinetic energy, and Vapor Volume Fraction occur between 0.5 and $1 \mathrm{~ms}$. In this interval of the injection process, the fuel mass flow rates are maximal. There is a significant fall in diesel fuel average normal velocity and a significant increase in the average value of turbulent kinetic energy at 40 bar of in-cylinder pressure. These fluctuations occur at the moment of the sudden rise of the Vapor Volume Fraction at the nozzle hole exit, which occurs around $0.5 \mathrm{~ms}$ after the injection start. There are also some fluctuations of the Vapor Volume Fraction for GTL fuel at the nozzle hole outlet, but these are significantly lower compared to the fluctuations of VVF for diesel fuel. This indicates that smaller values of cavitation structures at the nozzle hole outlet always have a smaller influence on the values of the normal fuel velocity and turbulent kinetic energy. When the fuel vapor structures at nozzle outlet exceed a certain value (amount), they influence fuel average normal velocity, and the average value of turbulent kinetic energy becomes significant. Because the diesel fuel mass flow does not drop at the same time as the fuel normal velocity, we believe that the occurrence of Vapor Volume Fraction might cause the formation of eddies in the fuel flow, which increases other fuel velocity components and further increases values of turbulent kinetic energy.

\section{Conclusions}

In the present paper, 1D and 3D numerical simulations were performed to study the influence of the physical-chemical properties of diesel and GTL fuels on the injection process, in-nozzle flow, and cavitation inception in a solenoid injector of a common-rail injection system. The obtained influences are:

- Higher average mass flow rates and velocities were obtained for the diesel fuel injection process. This behavior can be associated with the higher diesel fuel density and lower viscosity compared to GTL fuel. Higher mass flow rates of diesel fuel can also be connected to larger injected diesel fuel mass.

- The cavitation in the injection hole is triggered regardless of the differences in fuel properties. It occurs in the upper part of the injection hole entry for both fuels tested and spreads through the injection hole towards the hole exit. The difference in fuel properties influences the number of formed cavitation structures. The higher viscosity and lower saturation pressure of GTL fuel leads to slightly smaller areas of cavitation compared to diesel fuel.

- The occurrence of cavitation in the injection hole is highly influenced by the difference between injection and in-cylinder back pressure. Higher pressure differences produce larger areas of cavitation fraction and vice versa.

- The occurrence of cavitation (Vapor Volume Fraction) at the injection hole exit influences the effective flow area, which strongly influences the velocity at the hole exit. When a large amount of cavitation is present at the hole exit, fluctuations in flow velocity are obtained. The observed phenomenon is more significant when the difference between injection and in-cylinder back pressure is high.

- The obtained results indicate that the appearance of Vapor Volume Fraction at nozzle exit influence the magnitude of turbulent kinetic energy. This phenomenon is also more significant at higher differences between injection pressure and in-cylinder back pressure.

- The appearance of Vapor Volume Fraction at the nozzle outlet might cause the formation of eddies in fuel flow, which further effects the magnitude of each fuel velocity component.

The authors believe that the observed differences at the injection hole exit can have a significant and positive impact on fuel atomization as a means of better fuel disintegration and larger spray cone angles. This can usually lead to enhanced fuel evaporation and better, cleaner combustion. According to our previous study [21], cavitation at the injection hole exit can influence the center axis of fuel spray cone, which can decline. This can cause 
the formation of asymmetrical fuel spray and further increase the possibility of two fuel spray collisions, which will have a negative impact on fuel atomization. The collision of sprays can cause the fuel droplets to merge and make them harder to evaporate, which can lead to poor combustion and increase the formation of harmful emissions.

Overall, the presented results indicate that the GTL fuel can be used as a substitute fuel in the modern common-rail diesel engines. However, the influence of GTL properties on fuel spray development, emission formation, etc., must be studied before its integration.

Finally, the agreement between experimental and numerical results confirms the suitability of the used numerical procedure to study the effect of fuel properties in a common-rail injection nozzle.

Author Contributions: All authors have contributed equally to the development of this document. All authors have read and agreed to the published version of the manuscript.

Funding: The Spanish Ministry of Science, Innovation and Universities under Research Project "RECOVER" ref. RTI2018-095923-B-C21, and Castilla La Mancha Regional Government under Research Project "ASUAV" ref. SBPLY/19/180501/000116, have supported this work.

Data Availability Statement: Not applicable.

Acknowledgments: The authors wish to thank AVL LIST GmbH for their support by providing the AVL software program used during the research.

Conflicts of Interest: The authors declare no conflict of interest.

\section{References}

1. Eurostat. Energy Balance Sheets 2016 Data; European Union: Luxembourg, 2018. [CrossRef]

2. EEA. Emissions of Air Pollutants from Transport; European Environment Agency: Copenhagen, Denmark, 2018.

3. Lešnik, L.; Biluš, I. The effect of rapeseed oil biodiesel fuel on combustion, performance, and the emission formation process within a heavy-duty DI diesel engine. Energy Convers. Manag. 2016, 109, 140-152. [CrossRef]

4. Küüt, A.; Ilves, R.; Küüt, K.; Raide, V.; Ritslaid, K.; Olt, J. Influence of European union Directives on the Use of Liquid Biofuel in the Transport Sector. Procedia Eng. 2017, 187, 30-39. [CrossRef]

5. European Commission Report. Renewable Energy Progress Report; European Commission: Brussels, Belgium, 2019.

6. Alalwan, H.A.; Alminshid, A.H.; Aljaafari, A.S. Promising evolution of biofuel generations. Subject review. Renew. Energy Focus 2019, 28, 127-139. [CrossRef]

7. Mujtaba, I.; Srinivasan, R.; Elbashir, N. The Water-Food-Energy Nexus; CRC Press: Boca Raton, FL, USA, 2018. [CrossRef]

8. Ezzitouni, S.; Soriano, J.A.; Gómez, A.; Armas, O. Impact of injection strategy and GTL fuels on combustion process and performance under diesel engine start. Fuel 2017, 200, 529-544. [CrossRef]

9. Li, T.X.; Zhu, D.L.; Akafuah, N.K.; Saito, K.; Law, C.K. Synthesis, droplet combustion, and sooting characteristics of biodiesel produced from waste vegetable oils. Proc. Combust. Inst. 2011, 33, 2039-2046. [CrossRef]

10. Battisoni, M.; Magnotti, G.; Genzale, C.; Arienti, M.; Matusik, K.E.; Duke, D.J. Experimental and Computational Investigation of Subcritical Near-Nozzle Spray Structure and Primary Atomization in the Engine Combustion Network Spray D. SAE Int. J. Fuels Lubr. 2018, 11, 337-352. [CrossRef]

11. Manin, J.; Pickett, L.M.; Yasutomi, K. Transient cavitation in transparent diesel injectors, ICLASS 2018. In Proceedings of the 14th Triennial International Conference on Liquid Atomization and Spray Systems, Chicago, IL, USA, 22-26 July 2018.

12. Battistoni, M.; Som, S.; Powell, C.F. Highly resolved Eulerian simulations of fuel spray transients in single and multi-hole injecotrs: Nozzle flow and near-exit dynamics. Fuel 2019, 251, 709-729. [CrossRef]

13. Bicer, B.; Sou, A. Application of the improved cavitation model to turbulent cavitation flow in fuel injector nozzle. Appl. Math. Model. 2016, 40, 4712-4726. [CrossRef]

14. He, Z.; Shao, Z.; Wang, Q.; Zhong, W.; Tao, X. Experimental study of cavitating flow inside vertical multi-hole nozzles with different length-diameter ration using diesel and biodiesel. Exp. Therm. Fluid Sci. 2015, 60, 252-262. [CrossRef]

15. Srinivasan, V.; Salazar, A.J.; Saito, K. Modeling the disintegration of cavitating turbulent liquid jets using a novel VOF-CIMD approach. Chem. Eng. Sci. 2010, 65, 2782-2796. [CrossRef]

16. Srinivasan, V.; Salazar, A.J.; Saito, K. Numerical simulation of cavitation dynamics using a cavitation-induced-momentum-defect (CIMD) correction approach. Appl. Math. Model. 2009, 33, 1529-1559. [CrossRef]

17. Flores-Herrera, L.A.; Sandoval-Pineda, J.M.; Silva-Rivera, U.S.; Tamayo-Meza, P.A.; Rivera-Blas, R. CFD Simulation of Obstructed Ventilation Ports in a Subway Tunnel Section. Int. J. Simul. Model. 2017, 16, 386-498. [CrossRef]

18. Andriotis, A.; Gavaises, M.; Arciumanis, C. Vortex flow and cavitation in diesel injector nozzles. J. Fluid Mech. 2008, 610, 195-215. [CrossRef] 
19. Gavaises, M.; Andriotis, A.; Papoulias, D.; Mitroglou, N.; Theodorakakos, A. Characterization of string cavitation in large-scale diesel nozzles with tarped holes. Phys. Fluids 2009, 21, 052107. [CrossRef]

20. Moon, S.; Huang, W.; Wang, J. First observation and characterization of vortex flow in steel micronozzles for high-pressure diesel injection. Exp. Therm. Fluid Sci. 2019, 105, 342-348. [CrossRef]

21. Lešnik, L.; Kegl, B.; Bombek, G.; Hočevar, M.; Biluš, I. The influence of in-nozzle cavitation on flow characteristics and spray break-up. Fuel 2018, 222, 550-560. [CrossRef]

22. Margot, X.; Garcia, A.; Fajardo, P.; Patouna, S. Analysiy of the cavitation flow in real size diesel injectors with fixed and moving needle lift simulations. In Proceedings of the V European Conference on Computational Fluid Dynamic ECCOMAS CFD, Lisbon, Portugal, 14-17 June 2010.

23. Battistoni, M.; Grimaldi, C.N. Numerical analysis of injector flow and spray characteristics from diesel injector using fossil and biodiesel fuels. Appl. Energy 2012, 97, 656-666. [CrossRef]

24. Dai, X.; Wang, Z.; Liu, F.; Wang, C.; Sun, C.; Xu, C. Simulation of throttling effect on cavitation for nozzle internal flow. Fuel 2019, 243, 277-287. [CrossRef]

25. Xiao, M.; Zhang, W.; Shi, J. Numerical investigation of cavitation in a nozzle on the LNG fuel internal flow characteristics. In Proceedings of the First International Conference on Information Sciences, Machinery, Materials and Energy, ICISMME, Chongqing, China, 11-13 April 2015; pp. 1767-1771.

26. Shrivastava, P.; Verma, T.N. Effect of fuel injection pressure on the characteristics of CI engine fuelled with biodiesel from Roselle oil. Fuel 2020, 265, 117005. [CrossRef]

27. Ge, J.C.; Kim, H.Y.; Yoon, S.K.; Choi, N.J. Optimization of palm oil biodiesel blends and engine operating parameters to improve performance and PM morphology in a common rail direct injection diesel engine. Fuel 2020, 260, 116326. [CrossRef]

28. Yu, W.; Yang, W.; Zhao, F. Investigation of internal nozzle flow, spray and combustion characteristics fueled with diesel, gasoline and wide distillation fuel (WDF) based on a piezoelectric injector and a direct injection compression ignition engine. Appl. Therm. Eng. 2017, 114, 905-920. [CrossRef]

29. Torelli, R.; Soma, S.; Pei, Y.; Zhang, Y.; Traver, M. Influence of fuel properties on internal nozzle flow development in a multi-hole diesel injector. Fuel 2017, 204, 171-184. [CrossRef]

30. Jiang, G.; Zhang, Y.; Wen, H.; Xiao, G. Study of the generated density of cavitation inside diesel nozzle using different fuels and nozzles. Energy Convers. Manag. 2015, 103, 208-2017. [CrossRef]

31. Soriano, J.A.; Mata, C.; Armas, O.; Avila, C. A zero-dimensional model to simulate injection rate from first generation common rail diesel injector under thermodynamic diagnosis. Energy 2018, 158, 845-858. [CrossRef]

32. Soriano, J.A.; Garcia-Contreras, R.; Leiva-Candia, D.; Soto, F. Influence on Performance and Emissions of an Automotive Diesel Engine Fueled with Biodiesel and Paraffinic Fuels: GTL and Biojet Fuels Farnesane. Energy Fuels 2018, 32, 5125-5133. [CrossRef]

33. AVL List GmbH. AVL BOOST Hydsim Users Guide; AVL List GmbH: Graz, Austria, 2013.

34. Wang, C.; Moro, A.; Xue, F.; Wu, X.; Luo, F. The influence of eccentric needle movement on internal flow and injection characteristics of a multi-hole diesel nozzle. Int. J. Heat Mass Transf. 2018, 117, 818-834. [CrossRef]

35. AVL List GmbH. AVL Eulerian Multiphase Theory; AVL List GmbH: Graz, Austria, 2015.

36. Wang, X.; Li, K.; Su, W. Experimental and numerical investigation on internal flow characteristics of diesel nozzle under real injection conditions. Exp. Therm. Fluid. Sci. 2012, 42, 7718-7733. [CrossRef]

37. Saleh, B.; Ezz El-Deen, A.; Ahmed, S.M. Effect of liquid viscosity on cavitation damage based on analysis of erosion particles. J. Eng. Sci. 2011, 39, 327-336. [CrossRef]

38. Nouri, J.M.; Mackenzie, S.; Gaskell, C.; Dhunput, A. Effect of viscosity, temperature and nozzle length-to-diameter ration on internal flow and cavitation in a multi-hole injector. Fuel Syst. IC Engines 2012, 265-278. [CrossRef] 